\title{
Multiverse Explanation for Small Higgs Mass
}

\section{A new model that assumes that a multitude of universes existed when our Universe first formed may explain why the Higgs mass is smaller than traditional models predict.}

By Katherine Wright

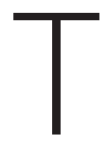
he standard model of particle physics has been inordinately successful, providing experimental predictions that accurately describe most of our Universe's forces and fundamental particles. But the model has some glaring holes. It contains no viable dark matter particle, it lacks an explanation for the Universe's accelerating expansion, and it predicts a Higgs boson mass that is at least triple that measured in experiments. Now, Raffaele Tito D'Agnolo of the University of Paris-Saclay and Daniele Teresi of CERN have developed a model that provides an explanation for the lightness of the Higgs [1]. The duo says that their model, which invokes multiverses, could also explain other conundrums, such as the observed similarity in how matter and antimatter experience strong interactions.

As a starting point for their model, the researchers assume that at very early times in our Universe's history there existed a multitude of universes. Each universe contained Higgs bosons

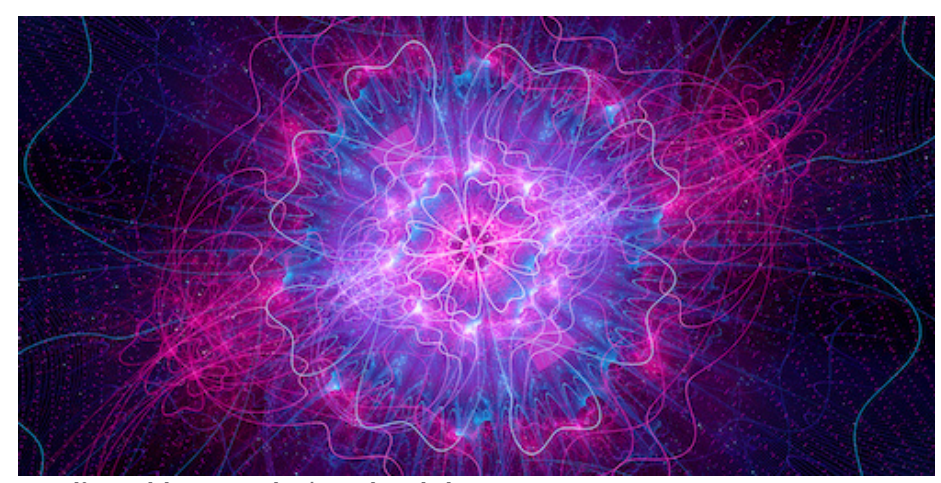

Credit: sakkmesterke/stock.adobe.com with inhomogeneous masses: some regions of each universe contained a heavy Higgs boson, while others contained a very light version.

By watching how this so-called multiverse evolved over time, the researchers found that regions having a large Higgs were unstable and collapsed in times as short as $10^{-5} \mathrm{~s}$. That collapse, also known as crunching, destroyed those multiverse components. The only remaining universe-ours-contained a very light Higgs boson. D’Agnolo and Teresi also found another factor in their model that prevented this universe from being crunched: a symmetric strong interaction-a fundamental force of nature that occurs between subatomic particles-for matter and antimatter. This symmetry is another hole of the standard model. The team says that their model should be testable in future dark matter and hadronic experiments.

Katherine Wright is the Deputy Editor of Physics.

\section{REFERENCES}

1. R. T. D’Agnolo and D. Teresi, "Sliding naturalness: New solution to the strong-CP and electroweak-hierarchy problems," Phys. Rev. Lett. 128, 021803 (2022). 